\title{
Avaliação dos serviços hospitalares de emergência do estado do Rio de Janeiro
}

\author{
Evaluation of emergency hospital services \\ in the state of Rio de Janeiro
}

\author{
Gisele O 'D wyer ${ }^{1}$ \\ I sabela Escórcio Augusto da M atta ${ }^{1}$ \\ Vera Lucia Edais Pepe ${ }^{1}$
}

${ }^{1}$ Departamento de Administração e Planejamento em Saúde, Escola Nacional deSaúde Pública, Fiocruz. Rua Leopoldo Bulhões 1.489/

728, M anguinhos.

21041-210 Rio de Janeiro

RJ. odwyer@ensp.fiocruz.br
Abstract This study aimed at the evaluation of urgency/emergency hospital units and recorded the relations of emergency units with users, other hospital services and the healthcare network and approached the size of the teams, the relations among the professionals, their qualification and income. It further describes the infrastructure of these services including basic organizational aspects. The aspect care was one component in this broader object. It is a descriptivestudy applying a questionnaire to the heads of the emergency services of a sample of 30 hospitals. The statements suggest the overcrowding of emergency services (in $80 \%$ of hospitals) deteriorating the work and care conditions to be due to the inefficiency of primary care and the healthcare network. The deficit of human resources was found larger than the lack of technology. The precarious employment relations were considered a factor hampering the qualification and retention of professionals. The most favorably evaluated items were: re lationship with service heads, trust and expectations with regard to the service. The worst items were: wages, team size and labor medicine. The nvestment in human resources is indispensable. There is a need for public policies that articulate more efficient interventions given that those in force are defining the configuration of territories with their hierarchized and solidary networks. Key words Emergency, Evaluation, Hospital quality
Resumo 0 presente estudo avalia as U nidades H ospitalares de U rgência/Emergência e registrou a relação da emergência com usuários, outros serviços do hospital e com a rede assistencial. Abordou a relação entre trabalhadores, a dimensão do seu quadro, formação e vencimentos. Relacionou a infra-estrutura cobrindo aspectos essenciais da organização, onde a assistência foi um dos componentes desse objeto mais amplo. Foi realizado um estudo descritivo com aplicação de questionário para os chefes de serviços, em uma amostra de 30 hospitais. Os depoimentos indicam que a ineficiência da atenção básica e da rede induzem à superlotação (que atingiu $80 \%$ dos hospitais), o que deteriora as condições de trabal ho e assistência. 0 déficit de recursos humanos encontrado foi maior que o tecnológico. A precariedade dos vínculos empregatícios foi apontada como fator que dificulta a fixação e qualificação do profissional. Os itensmelhor avaliadosforam: relacionamento com chefia, confiança e expectativa com o serviço. Os piores foram salários, tamanho de equipe e medicina do trabalho. 0 investimento em recursos humanos é imprescindível. Há necessidade das políticas públicas articularem intervenções mais efetivas já queas vigentes definem a configuração dos territórios sanitários com suas redes hierarquizadas e solidárias.

Palavras-chave Emergência, Avaliação, Qualidade hospitalar 
Introdução

Analisando o SUS a partir desuas diretrizes, constata-se que existe um descompasso entre a conquista de direitos pela população e as reais e insuficientes condições para garanti-los. Porém, é inegável que ele aumentou a sua partici pação no financiamento dos serviços realizados no país, principalmenteem favor das populações de mais bai xa renda ${ }^{1}$. Os prontos-socorros ainda são importante porta de entrada para assi stência médica e boa parte da população usuária do SU S busca assistência por meio de consultas de pronto atendimento nos prontos-socorros dos hospitais, em vez de buscar esse tipo de atendimento na rede básica de saúde (porta de entrada presumida). É fato que o padrão deutilização deserviços de saúde de um determinado grupo populacional é principalmente explicado por seu perfil de necessidade em saúde²; porém, as preferências eescol has dos usuários influenciam na utilização dos serviços tanto quanto a forma como a rede está estruturada. Essa constatação precisa ser incorporada por gestores e profissionais para diminuir a expectativa de que a resolução estrutural da rede de serviços de saúde é condição suficiente para corrigir o inadequado padrão de utilização dos mesmos.

A Pesquisa Nacional deAmostragem D omiciliar (PNAD) $2003^{1,3}$ mostra a complexidade do SUS, seus avanços, sua iniqüidade, sua capacidade de oferta de serviços. Atualmente, a atenção às urgências está centrada nos hospitais. Os serviços de emergência funcionam com grande afluxo de demanda espontânea, culminando com a superlotação e, conseqüentemente, com a percebida baixa qualidade da assistência. 0 acesso aos recursos do SU Sé difícil, as filas de espera para consultas, exames e cirurgias são longas e faltam vagas para internação, bem como pessoal capacitado.

É interessante ressaltar que o grande acúmulo de doentes nos serviços de emergência ocorre tanto no setor público como no privado, tanto no Brasil ${ }^{4}$ como no exterior, em países em desenvolvimento ou em grandes potências ${ }^{5-12}$.

Em situações de excesso de demanda, os pacientes que precisam de recursos tecnológicos sofisticados em função da gravidade ou complexidade do seu problema de saúde são alvo de maior preocupação dos profissionais, dos gestores e dos formuladores de políticas de saúde. Os pacientes que exigem menos recursos tecnológicos são absorvidos como secundários, atendidos rapidamente após uma triagem e não são, linearmente, os que necessitam de menos aten- ção. Deve-se evitar que esse paciente seja rotulado como "inapropriado" ao serviço e que sofra a frustração de ter seu atendimento negado como eventualmente tem sido sugerido pela literatu$\mathrm{ra}^{13}$. Restrições de acesso têm sido propostas ${ }^{14}$, mas não são aceitáveis em um país com tanta desigualdade social como o Brasil.

Além da dificuldade de absorção pelo próprio hospital do atendimento gerado na emergência, principalmente da urgência clínica, existe pouca conexão entre esse atendimento e o seguimento possível na rede. A emergência, por suas características, acaba fazendo o papel de reguladora do sistema, ou seja, depósito dos problemas não resolvidos.

As dificuldades que o hospital e a atenção básica sempre tiveram para aceitar elidar com a porta hospitalar deurgência e pronto atendimento são bem conhecidas. A percepção que se tem é que, se a atenção básica pudesse, livrar-se ia dos prontos atendimentos e o hospital, das suas portas hospitalares de urgência ${ }^{15}$.

Para além do diagnóstico dos maus resultados do SUS, exaustivamente apontados pela literatura, pelas corporações profissionais e pela imprensa, estratégias deenfrentamento têm sido propostas pelo Estado através de políticas de saúde.

A formulação de política tem que ser entendida essencialmente como um processo político, em vez de um processo analítico de solução de problema. É um processo de negociação, barganha e acomodação de muitos diferentes interesses que refletem a ideologia do governo em poder ${ }^{16}$.

Uma proposta importante para as grandes emergências foi o QualiSU ${ }^{17}$, um conjunto de mudanças que visa proporcionar maior conforto para o usuário, atendimento de acordo com 0 grau de risco, atenção mais efetiva pelos profissionais de saúde e menor tempo de permanência no hospital. Além de uma reforma mais estrutural dos hospitais, é criado um grupo de humanização do atendimento e há uma reorganização da oferta de leitos com priorização para a emergência, rel evante porta de entrada para o sistema hospitalar. Concomitante ao QualiSUS, outras políticas que visavam à melhoria da qualidade da assistência foram implantadas, como a Política N acional de H umanização e a criação deCentrais de Regulação de Leitos, estratégica para a emergência. A ordenação do acesso à rede assistencial de urgência por meio da regulação médica pode transformar o cenário dos hospitais de referência em urgência einduzi r a reorganização, com o aumento da resolutividade e da qualidade dos serviços ${ }^{18}$. 
Outra importante conquista foi a Implantação do Serviço deAtendimento M óvel deUrgência (SAM U/192), um serviço desocorro pré-hospitalar móvel, que permitea organização no acesso à emergência e a racionalização da utilização da rede pública.

Ao mesmo tempo, normas legais ${ }^{19}$ transferem formalmente, para o Programa de Saúde da Família (PSF), a responsabilidade de atender à pequena urgência, prática ainda não disseminada nesses serviços.

A integração de serviços por meio de redes assistenciais remete ao conceito de integralidade que reconhece a interdependência dos atores e organizações, em face da constatação de que ne nhuma delas dispõe de recursos e competências necessários para a solução dos problemas de saúde de uma população ${ }^{20}$. A integração consiste em coordenar as organizações autônomas para a realização de um projeto coletivo comum. Os discursos e as políticas sobre a integração fundamentam-se, assim, em três linguagens: a estrutural (modificação das fronteiras das organizações), a clínica (modificações nas práticas profissionais) e a da cooperação (novos formatos denegociação e de acordos entre atores e organizações ${ }^{20}$. A instituição da rede exige estrutura organizacional, mas também exige modificação nos processos de trabalho e envolvimento cognitivo e afetivo dos profissionais.

Reforçando as políticas, existem movimentos de qualificação das práticas, vide HumanizaSUS (PNH, vinculada à Secretaria de Atenção à Saúde - SAS/M S, 2003), cartilha do usuário ${ }^{21}$, controle social no SU S22, etc.

Teoricamente, as políticas de saúde orientam as práticas, mas fatores sobre os quais os propositores de políticas não (ou pouco) interferem fazem com que elas aconteçam ou não. 0 processo de implementação envolve a interação entre a comunidade e o serviço de saúde, assim como entre o provedor e o receptor. Cuidado de baixa qualidade, atitudes rudes, falta de comunicação, proble mas de incentivos de recursos humanos, motivação, suporteesupervisão, podem todosintervir para fazer uma implementação de sucesso, ou levar a perder a oportunidade para intervenção $0^{16}$.

Portanto, o cenário que se pretendediscutir é de uma prática extremamente normativa (que estabelece portas de entrada e mecanismos rígidos de referência e contra-referência), quesilencia o pacienteequenão dá conta decumprir com os preceitos dos direitos universais à saúde e menos ainda com os de integralidade. É importante frisar que o não cumprimento desses pre- ceitos não pode ser unicamente atribuído à prática assistencial, já que existe uma insuficiência estrutural de serviços. 0 subfinanciamento éum impeditivo concreto de realização de um SUS mais justo, mas o enfrentamento de problemas não estruturais exige mais do que políticas de Estado. Exige reflexão e principalmente maior engajamento de diversos atores. Os profissionais de saúde são os vilões? N ão. São formados tendo como referência natural eclassificado como padrão de normalidade o secular processo de exclusão23. Daí a importância de ouvi-los para estudar a realidade dos serviços.

Deve-se entender a prática dos serviços como um processo social, sem deixar de atribuir ao profissional capacidade de reflexão, inovação esuperação, já que o SUS é repleto de experiências bemsucedidas de encontros solidários entre profissionais e usuários. Portanto, pretendeu-se desvendar o processo social que determina práticas e compreender o papel do agentehumano. Para o estudo das práticas sociais, Giddens ${ }^{24}$, a partir da Teoria da Estruturação, defendeque as práticas institucionalizadas estão profundamente enraizadasno tempo e no espaço. A vida social, em muitos aspectos, não éum produto intencional de seus atores constituintes, embora a conduta no dia- dia seja cronicamente levada a cabo de modo deliberado ${ }^{24}$.

Concebemos a nossa prática como baseada em um saber científico, objetivo, racional e, portanto, neutro. $\mathrm{Na}$ verdade, a prática é determinada por relações sociais, por valores construídos socialmente e deve ser reflexiva e crítica. As razões são causas de atividades que 0 indivíduo "faz acontecer" como característica inerente a ele ser um agente. [...] $M$ as existem fatores causais influenciando a ação sem atuar através da racionalização ${ }^{24}$.

Essas influências são as mais importantes, segundo Giddens, porque existem elementos nos contextos sobre os quais os atores não têm controle. São características facilitadoras ou coercivas do contexto.

A Teoria da Estruturação trata da dualidade que existe entre os sistemas sociais e as ações do agente humano. A ação nessa pesquisa estaria representada pelas práticas investigadas nos serviços e a estrutura seria o próprio serviço de emergência hospitalar na organização do SUS.

\section{A Vigilância Sanitária e os serviços de emergência}

A origem dos problemas sanitários sobre os quais 
a vigilância deve intervir para eliminar, diminuir ou prevenir riscos à saúde, inclui o meio ambiente, a produção e circulação de bens e a prestação de serviços de interesse à saúde ${ }^{25}$. 0 monitoramento da qualidade dos serviços de saúde é uma ação primordial para a vigilância sanitária e desde 2004 foi pactuado que os serviços hospitalares deemergência seriam inspecionados anualmente. 0 roteiro utilizado para a inspeção sanitária éum roteiro que trata da estrutura do serviço eretrata muito pouco do processo de trabalho.

O Centro de Vigilância Sanitária da Secretaria Estadual de Saúde do Estado do Rio de Janeiro (CVS-SES-RJ) financiou este estudo através de um Convênio de Cooperação Técnica, Pesquisa e Comunicação em Vigilância Sanitária, realizado entre o CVS-SES-RJ e a Escola N acional de Saúde Pública Sergio Arouca (ENSP), por reconhecer a complexidade e a importância do serviço de emergência no resultado dos indicadores de morbi-mortalidade da população. 0 objetivo foi de contribuir para a elaboração de uma metodologia de avaliação dos serviços de emergência hospital ar (edo respectivo instrumento de avaliação) que contemplasse a sua dimensão sistêmica.

\section{Metodologia}

A opção metodológica foi avaliar o serviço a partir do olhar de atores importantes nas emergências: o chefe da emergência; o chefe da enfermagem da emergência e o chefe médico de plantão. 0 estudo caracterizou-se como um estudo de caso, exploratório edescritivo com aplicação de questionário.

Para a seleção dos tópicos que compuseram o questionário, foram revisados: a legislação sanitária; os instrumentos de avaliação do Programa Nacional de Avaliação de Serviços de Saúde (PNASS); os roteiros de inspeção da vigilância sanitária e o M anual Brasilei ro de Acreditação. A partir destes instrumentos, construiu-se um novo questionário que foi submetido a um painel de especialistas para validação.

0 questionário foi dividido em cinco partes. A primeira referiu-se às características da clientela. Foram avaliados a abrangência geográfica de origem da clientela, a lotação da emergência e seus determinantes, a estratégia de gerência para a superlotação e se existe regulação de leitos. A segunda parte do roteiro tratou dos recursos tecnológicos e humanos da emergência. Foi analisada a pertinência de recursos para a missão pre- tendida pelo serviço. $\mathrm{Na}$ terceira parte, foram descritos a porta de entrada, o relacionamento com os serviços internos e externos ao hospital que interferem com a emergência e o tipo de doença ou condição clínica que o sistema tem mais dificuldade de absorver. A quarta tratou de resultados de taxa de ocupação, tempo de permanência e de internação. $E$, finalmente, o último bloco tratou da avaliação do serviço sob o ponto de vista do entrevistado.

Para selecionar as unidades investigadas, foi definido o universo de 119 serviços hospitalares de emergência do estado do Rio de Janeiro (dados do Cadastro Nacional de Estabelecimentos de Saúde - CNES - em 2005) e construída uma amostra. 0 tamanho da amostra foi calculado através do modelo de amostragem al eatória simples ${ }^{26}$, utilizando como parâmetroso nível designificância de 5\%, a proporção máxima de 50\% e margem de erro de $16 \%{ }^{26}$, obtendo, então, um total de trinta unidades de saúde. Tratando-se de uma amostragem estratificada, o método de alocação da amostra foi realizado através da partiIha proporcional que consistiu em calcular o tamanho da amostra proporcionalmente ao número de estabelecimentos de saúde para cada região do município. 0 critério utilizado para sel eção dos hospitais de cada região foi o de maior número de leitos. Esta escolha visava manter a comparabilidade com os hospitais do município do Rio de Janeiro do Programa QualiSUS. Foi substituído um hospital, na região N oroeste fluminense, por recusa do diretor em participar. A pesquisa de campo começou em dezembro de 2005 eterminou em junho de 2006. A maior parte das respostas foi tratada estatisticamente. A partir das respostas abertas, foi possível identificar categorias que foram utilizadas para a descrição e análise dessas questões, sistematizando as diversas maneiras com as quais os profissionais se expressaram.

O estudo foi submetido ao Comitê de Ética em Pesquisa da Escola Nacional de Saúde Pública Sergio Arouca/ Fundação Oswaldo Cruz (CAAE 0129.0.031.000-05) eaprovado no dia 14/09/2005.

\section{Resultados}

Dos hospitais visitados, 37\% ( $n=11)$ tinham mais de duzentos leitos (dados colhidos do CNES), $33 \%(n=10)$ tinham entre cem eduzentos leitose $30 \%(n=9)$ menos que 100 leitos, sendo que desses, apenas um tinha menos que cinqüenta leitos. 


\section{As emergências superlotadas}

Em todas as regiões do estado, havia superlotação deemergências, sendo que em cinco regiões todos os serviços visitados estavam constantemente superlotados. Nas outras cinco regiões, as emergências eram parcialmente superlotadas. Em números absolutos, apenas $20 \%$ dos hospitais $(n=6)$ negaram superlotação. A seguir, apre sentamos um mapa demonstrando o estado dividido pelas regiões estudadas, apontando onde existia superlotação plena e onde ela era parcial. O mapa nos dá a impressão de que o estado foi dividido em duas partes, uma mais litorânea e com emergências superlotadas e outra mais interiorana com emergências parcialmente lotadas.

A principal causa desuperlotação nas 24 emergências, apontada por todos os entrevistados, foi 0 atendimento a pacientes com problemas ambulatoriais. Em $60 \%$ desses $(n=14)$, houve referência espontânea à falência da rede básica como determinante da superlotação. Em uma das regiões, onde estão situados três desses hospitais, existiam postos de saúde abertos 24 horas.

A abrangência da origem geográfica dos pacientes foi apontada como causa de superlotação em $54 \%$ hospitais $(n=13)$, a insuficiência da estrutura hospitalar regional, em $37 \%(n=9)$ e problemas sociais, em $12 \%(n=3)$. A pareceram como causas isoladas o desconhecimento da população sobre a missão do serviço de emergência e a confiança da população no hospital. Em um único hospital conveniado ao SUS, houve referência à má gestão do SUS e insuficiência de pagamento como causa de superlotação. Chamou a atenção, como outra causa apontada, a referência à problema de gestão interna em um hospital onde haviam leitos vagos e tempo de internação muito longo.

Nove hospitais, $37 \%$ dos 24 que apresentavam superlotação, não têm mecanismos de gerenciamento do problema. As iniciativas QualiSU S e a regulação de leitos ajudavam na gerência da superlotação de nove hospitais. Dois hospitais fecham as portas eventual mente como estratégia de reprimir a demanda e um outro restringe 0 atendimento. Um hospital, que conta com posto de saúde $24 \mathrm{~h}$ no seu município, transfere de ambulância os pacientes ambulatoriais. Outro hospital adota uma campanha informativa para a população para diminuir o número de atendimentos ambulatoriais na emergência (foIheto: "procure o hospital em caso de ..."). A chefia da emergência de um dos nove hospitais que referiram ajuda da regulação de leitos recusa pa- cientes que são ambulatoriais na emergência. Esse mesmo profissional exigia o cumprimento da carga horária de todos os médicos e acha que a médio prazo essa medida vai ajudar a gerenciar a superlotação. Dois hospitais remanejavam profissionais para gerenciar a superlotação. No quadro a seguir, são descritas as estratégias encontradas para gerenciar a superlotação.

Apenas seis hospitais não estavam vinculados a nenhuma Central de Regulação de Leitos. Cinco desses foram visitados em dezembro de 2005, quando começou a pesquisa de campo. 0 sexto referiu que, apesar do atraso burocrático da assinatura do convênio, utilizava informalmente a central, quando necessário. Em apenas $12 \%$ dos hospitais $(n=3)$, a regulação de leitos era atuante.

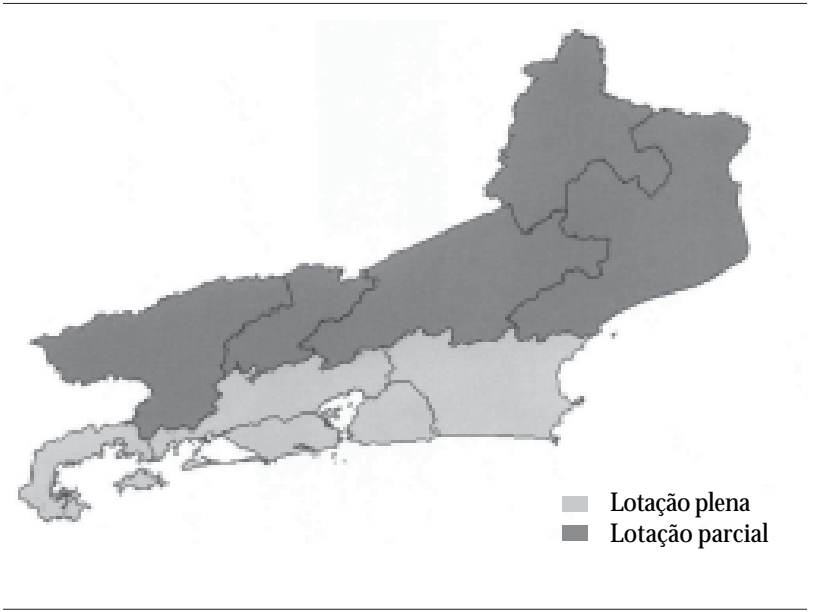

Figura 1. Lotação das emergências por região do estado.

Quadro 1. Estratégias descritas para gerenciar a superlotação.

\begin{tabular}{|c|l|}
\hline $\begin{array}{c}\text { Número de hospitais } \\
\text { (total =24) }\end{array}$ & \multicolumn{1}{|c|}{ Estratégia adotada } \\
\hline 9 & nenhuma \\
\hline 9 & $\begin{array}{l}\text { cumprir incentivos QualisuS } \\
\text { e regulação }\end{array}$ \\
\hline 2 & fechar as portas \\
\hline 2 & remanejar profissionais \\
\hline 1 & transferir para postos de saúde \\
\hline 1 & fazer campanha educativa \\
\hline
\end{tabular}


A seguir, o depoimento deum dos entrevistados que reflete as dificuldades de gerenciar uma emergência hospitalar superlotada.

A emergência não tem gestão sobre as vagas para internação. A média de permanência no hospital hoje é muito grande (dezessete dias). Falta motivação dos profissionais, não existe trabalho em equipe efalta organização das equipes. Os mé dicos da emergência não querem atender todos os casos porque são formados em especialidades diferentes. A unidade deveria contratar médicos emergencistas para emergência.

\section{As questões estruturais}

No Quadro 2, podem ser observados, por região, os resultados referentes aos recursos tecnológicos e humanos dos hospitais visitados.

Foi importante constatar que o déficit de recursos humanos era mais importante do que 0 de recursos tecnológicos. Em relação à estrutura,
$60 \%$ dos hospitais ( $n=18$ ) apresentavam suficiência em termos de recursos tecnológicos para suas atividades, sendo que dos seis hospitais do projeto QualiSUS apenas dois eram suficientes de recursos. É importante lembrar que o projeto QualiSUS pretende atingir as grandes emergências das capitais brasileiras e por isso beneficiou seis dos trinta hospitais estudados.

Os postos de trabalho foram questionados em relação à pertinência e suficiência para o serviço prestado. Portanto, quando houve indicação de falta de especialistas, tratava-se de serviço de referência, onde várias especialidades eram necessárias. 0 déficit de enfermagem foi maior do que o de médicos em geral, seguido pelo de especialistas. As capacitações questionadas foram em urgência e emergência por serem obrigatórias por legislação (Portaria M S n 2.048, de 5 de novembro de 2002). Os profissionais de enfermagem estavam mais capacitados e os recémcontratados eram o principal alvo de capacita-

Quadro 2. Resultados de estrutura de recursos tecnológicos e humanos, apresentados por região.

\begin{tabular}{|c|c|c|c|c|c|}
\hline Região & $\begin{array}{l}\text { Postos de } \\
\text { trabalho } \\
\text { completos }\end{array}$ & $\begin{array}{l}\text { Política de } \\
\text { educação } \\
\text { permanente }\end{array}$ & $\begin{array}{l}\text { Tipo de vínculo } \\
\text { empregatício }\end{array}$ & $\begin{array}{c}\text { Capacitação em } \\
\text { urgência/ } \\
\text { emergência }\end{array}$ & $\begin{array}{l}\text { Infra- } \\
\text { estrutura } \\
\text { suficiente }\end{array}$ \\
\hline Região 1 & não & ausente & diversos ${ }^{x}$ & parcial & não \\
\hline Região 2 & não & $\begin{array}{l}\text { parcialmente } \\
\text { implantada }\end{array}$ & $\begin{array}{c}\text { maioria } \\
\text { concursada }\end{array}$ & parcial & não \\
\hline Região 3 & não & $\begin{array}{l}\text { parcialmente } \\
\text { implantada }\end{array}$ & $\begin{array}{l}\text { concursados e } \\
\text { prestadores }\end{array}$ & parcial & não \\
\hline Região 4 & não & $\begin{array}{l}\text { parcialmente } \\
\text { implantada }\end{array}$ & diversos ${ }^{x}$ & parcial & sim \\
\hline Região 5 & não & $\begin{array}{l}\text { parcialmente } \\
\text { implantada }\end{array}$ & $\begin{array}{l}\text { concursados e } \\
\text { prestadores }\end{array}$ & parcial & $\operatorname{sim}$ \\
\hline Região 6 & $\operatorname{sim}$ & ausente & $\begin{array}{c}\text { maioria } \\
\text { prestadores }\end{array}$ & parcial & $\operatorname{sim}$ \\
\hline Região 7 & não & $\begin{array}{l}\text { parcialmente } \\
\text { implantada }\end{array}$ & $\begin{array}{l}\text { concursados e } \\
\text { prestadores }\end{array}$ & parcial & $\operatorname{sim}$ \\
\hline Região 8 & não & implantada & $\begin{array}{c}\text { maioria } \\
\text { prestadores }\end{array}$ & parcial & sim \\
\hline Região 9 & não & ausente & $\begin{array}{c}\text { maioria } \\
\text { prestadores }\end{array}$ & ausente & não \\
\hline Região 10 & sim & $\begin{array}{l}\text { parcialmente } \\
\text { implantada }\end{array}$ & diversos ${ }^{x}$ & $\begin{array}{l}\text { para todos os } \\
\text { médicos e } \\
\text { enfermeiros }\end{array}$ & $\operatorname{sim}$ \\
\hline
\end{tabular}

× Os vínculos foram considerados diversos quando havia vários tipos de contratos para os prestadores de serviços ou quando os profissionais eram concursados por unidades federativas diversas. 
ção, provavelmente para cumprir a legislação, apesar desses não serem os profissionais mais permanentes nos serviços. A variedade dos vínculos empregatícios apareceu como regra. Um hospital estava capacitando seus profissionais por iniciativa do QualiSUS. As políticas de educação permanente não estavam implantadas em 33\% dos hospitais ( $n=10)$ e parcialmente implantadas em $50 \%(n=15)$.

Segue 0 depoimento de um chefe da emergência, que caracteriza a fragilidade da situação dos recursos humanos nesses serviços.

A qualidadetécnica dosmeus médicos étão baixa que não sei como melhorar a resolutividade do serviço. Ainda bem que eles não agüentam ficar por muito tempo ea maioria tem contrato temporário.

\section{Processo de trabalho}

A primeira questão investigada foi a porta de entrada nessas emergências. Em todas as emergências visitadas, os pacientes graves são atendidos imediatamente. Seis hospitais, por terem pouco movimento, não achavam ser necessária a classificação de risco. Dos 24 superlotados, $29 \%$ $(n=7)$ trabal havam com equipes multiprofissionais na sala de espera eclassificação de risco, sendo que uma delas seguia os padrões do QualiSUS. Outra questão investigada foi como o serviço se relacionava com outros serviços do hospital e com outros serviços da rede. Oito referiram dificuldades internas, sendo que, em um deles, estavam sendo vencidas com o projeto QualiSUS. Seis referiram reserva de vagas e desnecessário prolongamento do tempo de internação, sendo que em três as dificuldades maiores eram com a clínica médica. No sétimo, havia um incentivo em forma de complementação salarial para os plantonistas, gerando um mal-estar junto aos profissionais da rotina. No oitavo serviço, houve uma mudança no critério de acesso dos pacientes, que antes privilegiava pedidos de políticos, gerando insatisfação para al guns profissionais. Questões coorporativas também foram citadas isoladamente.

As dificuldades externas foram apontadas em todos os hospitais. Os motivos apontados foram: insuficiência da rede básica; falta de vagas para internação na rede; falta de sintonia com 0 atendimento pré-hospitalar (SAMU e bombeiros); falta de vaga para real ização de exames complexos; indisponibilidade de ambulatórios especializados; falta de vagas em casa de apoio; problemas sociais. Foi encontrada, em uma emergência, uma situação muito irregular, de um pa- ciente "internado" há quatro anos. O utro aspecto verificado foi se, após a indicação de internação do paciente da emergência, na ausência de vaga, as clínicas solicitadas se co-responsabilizavam pela evolução do doente. Em seis hospitais, as diversas clínicas dividiam a responsabilidade pelo paciente. Em outros três, só os serviços cirúrgicos assumiam essa responsabilidade. Em três hospitais menores, os médicos da emergência assumem a responsabilidade pelo restante do hospital quando os profissionais da rotina não estão, o queéirregular, já que o médico da emergência deve ser exclusivo do serviço.

Em 53\% das emergências $(n=16)$, protocolos técnicos são utilizados regularmente.

Outra questão foi identificar quais os problemas mais difíceis de resolver e de encaminhar dentro da rede. Muitas doenças foram indicadas como difíceis de encaminhar para o sistema. M erecem nota pacientes crônicos, neurológicos, cirurgias ortopédicas, pacientes oncológicos, coronariopatas, tuberculosos, idosos em geral e pacientes com problemas sociais.

Indicadores hospitalares de desempenho

Foram identificados três cenários, como está representado no Quadro 3.

O primeiro cenário é composto por onze grandes emergências (37\%), onde as emergências têm taxa de ocupação de até $300 \%$. Muitos pacientes que necessitam de internação permanecem na emergência mais de 72 horas aguardando leito. Não é raro encontrar pacientes "internados" na emergência por dias. Dois desses hospitais só atendem emergência, não têm outra porta de entrada, e por isso o tempo de permanência na emergência tende a ser menor.

0 segundo grupo é composto por seis hospitais ( $20 \%$ ), onde de 10 a $25 \%$ dos pacientes permanecem na emergência por mais de 24 horas. 0 atendimento ocorre, na maioriadas vezes, em trinta minutos, mas a espera pode chegar a uma hora.

0 terceiro grupo é formado por 13 hospitais (43\%). N esses, os pacientes não ficam na emergência por mais deseis horas. Não há espera para 0 atendimento na emergência, sendo essa no máximo de trinta minutos. A taxa de ocupação da emergência fica em torno de $70 \%$ e a do hospital, $90 \%$. D ois desses hospitaischegaram a apresentar ocupação hospitalar de $50 \%$ dos leitos.

Infelizmente, em $26 \%$ dos hospitais ( $n=8$ ), as internações na clínica médicaultrapassavam trinta dias. Em um deles havia um paciente internado há um ano. 


\section{O pinião dos entrevistados}

Esta parte do questionário utilizou principalmenteos critérios presentes no PNASS. H ouvemuita variação de resposta neste bloco, mesmo entre os entrevistados de um mesmo hospital. 0 total das opiniões pode ser observado na Figura 2.

0 item melhor avaliado foi o relacionamento com a chefia, com $90 \%$ de "muito bom" e "satisfatório" ( $n=81$ de 90 entrevistados), seguido por confiança $(84 \%, n=76)$ eexpectativa com o serviço ( $76 \%$, $n=69$ ). Foi pior avaliado, com "muito ruim" e insatisfatório", o salário (69\%, $n=62)$, seguido pelo tamanho da equipe $(60 \%, n=54)$ e medicina do trabalho $(57 \%, \mathrm{n}=51)$.
Algumas particularidades merecem nota. Um único hospital avaliou negativamente todos os itens, com a exceção do relacionamento com a chefia. Outro hospital avaliou positivamente todos os itens. M etade dos entrevistados das emergências superlotadas têm uma expectativa sobre o serviço positiva, 0 que é importante para a adesão às mudanças necessárias. Sobre a confiança no serviço, seguem os depoimentos de dois chefes de serviços diferentes.

Se eu precisasse de atendimento de emergência, queria ser atendido aqui. Confio na equipee no serviço.

Quadro 3. Indicadores hospitalares agrupados por tipo de cenário.

\begin{tabular}{|c|c|c|c|c|}
\hline $\begin{array}{c}\text { No. de hospitais } \\
\text { (total de 30) }\end{array}$ & $\begin{array}{c}\text { Taxa de ocupação } \\
\text { da emergência }\end{array}$ & $\begin{array}{c}\text { Taxa de ocupação } \\
\text { do hospital }\end{array}$ & $\begin{array}{c}\text { Tempo de permanência } \\
\text { na emergência }\end{array}$ & $\begin{array}{c}\text { Tempo de espera } \\
\text { para o atendimento }\end{array}$ \\
\hline 11 & $100 \%$ & $100 \%$ & mais de $72 \mathrm{~h}$ & $3 \mathrm{~h}$ \\
\hline 6 & menos de $100 \%$ & até $100 \%$ & até $24 \mathrm{~h}$ & até $1 \mathrm{~h}$ \\
\hline 13 & até $6 \mathrm{~h}$ & até 30 min \\
\hline
\end{tabular}

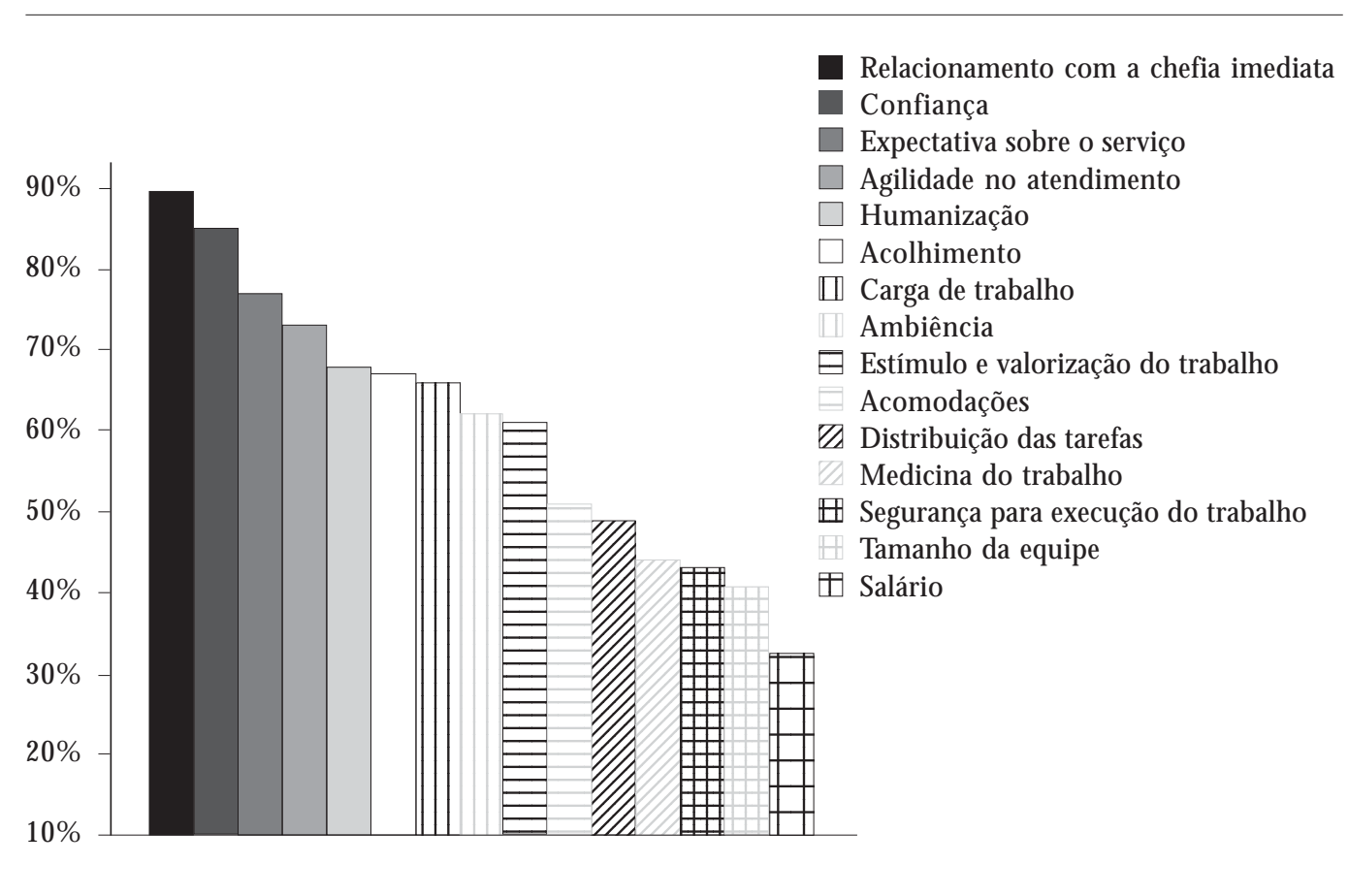

Figura 2. Percentual dos itens classificados como "muito bom" ou "satisfatório" pelos entrevistados. 
Apesar do caos do sistema de saúde, o hospital ainda consegue prestar um serviço de qualidade. A prefeitura tem investido no hospital, que é de referência para outros municípios.

Dez hospitais tiveram opiniões muito favoráveis (exceções: salário emedicina do trabalho).

A penas um hospital media e avaliava a satisfação e o desempenho dos trabalhadores. Existia - Programa de Incentivo e de Desempenho (PIDE), no qual cada funcionário recebe uma nota pelo seu desempenho e poderá receber adicional salarial de até $30 \%$. Em outros cinco hospitais, existia uma avaliação considerada incipiente. Os usuários tinham mais espaço para avaliação de sua opinião (referida em $40 \%$ dos hospitais, $n=12$ ). Essas avaliações eram feitas através de ouvidoria, caixa de sugestões e questionários ou entrevistas. Infelizmente, em um hospital conveniado, havia avaliação apenas para os pacientes particulares.

Em $16 \%$ dos hospitais ( $n=5)$, havia política de humanização implantada e, em 30\% (n=9), ela era incipiente. Em um hospital, a política produziu ações concretas de conforto para os usuários. Em outro, teve como principal impacto a sensibilização da equipe para os problemas internos do hospital, como pode ser visto no de poimento a seguir.

0 maisimportante hojeéa nossa inserção dentro da Política Nacional de Humanização e com 0 Grupo de Trabalho Humanização (GTH); estamos produzindo novas formas de compromisso através de um processo reflexivo e de conscientização dos funcionários em relação à percepção do hospital dentro da sua totalidade.

\section{Discussão}

Uma conseqüência da superlotação das emergências é a tendência restritiva. O utros estudos brasileiros sobre os serviços de emergência têm de monstrado a dificuldade de lidar com a demanda excessiva de pacientes não graves ${ }^{27}$ que se justifica por: fácil acessibilidade, funcionamento ininterrupto; impossibilidade de recorrer aos serviços ambulatoriais e também por se sentirem angustiadas e apreensivas ${ }^{28}$. 0 mesmo autor afirma que o excesso de demanda acarreta acúmulo de tarefas e conseqüentemente sobrecarga para toda a equipe de profissionais, contribuindo para o aumento dos custos hospitalares. Também aponta para a ineficácia do sistema de saúde, incapaz de oferecer a esses indivíduos um acesso maisfácil à rede de atenção primária ${ }^{28}$. A realida- de descrita nesse estudo é semelhante à encontrada na presente pesquisa, na qual o excesso de atendimentos ambulatoriais foi tido como a principal causa de superlotação.

Os serviços deveriam discutir com a rede como integrar esse tipo de paciente (o ambulatorial) às outras possíveis portas de entrada e preparar-se para atendê-lo, já que as demandas são geradas por fatores culturais e por deficiências de recursos tecnológicos e sociais. 0 depoimento de um entrevistado mostra que os profissionais reconhecem a necessidade de diálogo com a rede e aponta as dificuldades em travá-lo.

A direção vai aos Secretários, mas existe uma distância muito grande entre os funcionários e a Secretaria de Saúde.

Um outro chefe de serviço foi o único entrevistado que reconheceu a necessidade de acolher todas as demandas.

O hospital passa por mudanças grandes eradicais de filosofia de trabalho para modificar o atendimento e, portanto, não mais atenderá casos ambulatoriais. 0 que será feito com os pacientes que precisam de atendimento ambulatorial após a mudança?

Corresponder às expectativas da clientela é um dos maiores desafios para a organização da assistência ${ }^{29}$. Esse desafio deveser perseguido por gestores eacolhido pelos profissionais, já que são os atores da prática que legitimam o SU S perante a população.

Os postos de trabalho estavam incompletos em $80 \%$ dos trinta hospitais visitados $(n=24)$. Uma impressão obtida através das entrevistas é de que os profissionais que chefiam a emergência são experientes e conhecem a complexidade envolvida nesse atendimento. Em compensação, a existência de vários tipos de vínculos, alguns temporários, dificulta a fixação do profissional e aumenta a necessidade de investir na qualificação dos mesmos. Provavelmente, os vínculos são justificados por contratos emergenciais, mas precarizam a relação de trabalho, não resolvem a carência quantitativa de recursos humanos, diminuem o padrão qualitativo da equipe e não contribuem com a integração entreo corpo clínico da emergência e o hospital.

A insatisfação com a carga de trabalho e remuneração, apontadas pelos entrevistados (35\% e $69 \%$, respectivamente), relaciona-se com o tamanho e qualificação da equipe, mas também com o estresse inerenteao trabalho em emergência, agravado pelas condições insatisfatórias de saúde da população com a qual esses profissionais lidam. A insatisfação com o trabalho em 
emergência foi constatada em um estudo ${ }^{30}$ que acusa remuneração incompatível com o nível de exigência eresponsabilidade em um processo de trabal ho sistematicamenteatravessado pelas tensões pessoais, organizacionais e sociais. 0 depoimento a seguir ilustra a fragilidade do trabalhador da emergência.

São necessárias a modernização dos equipamentos, manutenção, política de RH com capacitação permanente e política de cargos e salários.

$N$ ão é admissível a falta de recursos materiais em um serviço deemergência, e o investimento deve ser estendido aos serviços ambulatoriais eaos de investigação diagnóstica, cuja assistência reflete na assistência hospitalar. Pesquisa recente do PNAD ${ }^{1,3}$ revela melhora nesse quadro, mas a insuficiência deleitos eletivos e de recursos diagnósticos ainda é restritiva.

A integração da rede foi avaliada nesteestudo através dos conflitos externos das emergências. As dificuldades giraram em torno da falta de vagas e da dificuldade de real ização de exames complexos. A Central de Regulação de Vagas é um dispositivo recente de otimização do acesso ao leito hospitalar. Em uma região, onde a pesquisa foi realizada em 2005, não houve referência à sua implementação. Nas outras nove regiões, visitadas em 2006, havia o convênio em oito delas, acenando para um cenário mais favorável.

A atenção básica foi alvo de muita queixa. A penasuma região não fez referências negativasà mesma. Com a melhor definição da rede e fortalecimento do PSF, pode ser que os serviços de emergência deixem de ser a real e principal porta de entrada do sistema e que o processo de trabaIho nesses serviços seja mais compatível com a sua missão.
A ouvidoria foi a forma de avaliação de satisfação de usuários mais encontrada; entretanto, outras estratégias são necessárias para possibilitar o controle social. Constatamos diferenças de tratamento de pacientes SUS em dois hospitais conveniados, o que fere os direitos constitucionais dos pacientes. 0 depoimento a seguir reflete a complexidade e gravidade do problema.

Enquanto não forem melhoradas as condições de trabalho e salários, não vai haver melhoria do atendimento prestado para o paciente do Sistema Único de Saúde.

O serviço hospitalar de emergência continua sendo um desafio para o SUS por ser complexo em termos de necessidades tecnológicas, por exigir um processo de trabalho árduo e por refletir as precárias condições sociais da população atendida e a precariedade da situação dos trabalhadores de saúde. A organização da rede éo caminho, assim como o controle social, a formação de recursos humanos e o financiamento compatível com as atribuições e responsabilidades dos serviços.

Constatou-se, como dado muito positivo, que a grande emergência recebe um tratamento de boa qual idade eque responde às necessidades dos pacientes mais graves, refletindo positivamente nos indicadores de morbidade e mortalidade. M as o pior atendimento prestado ao grupo de pacientes menos graves não atende aos princípios da eqüidade e da integralidade, além de perpetuar uma situação de precariedade da assistência. Garantir acesso a serviços de qualidade para todos é uma meta que os gestores do SUS, a Vigilância Sanitária e todos os profissionais de saúde devem incorporar como sua. 


\section{Colaboradores}

G O 'D wyer trabalhou na concepção, metodologia, pesquisa, análise dos dados, e redação do artigo; IEA M atta trabalhou na pesquisa, análise dos dados e redação do artigo eVLE Pepe trabaIhou na negociação junto ao CVS/SES-RJ da proposta de trabalho e na redação do artigo.

\section{Agradecimentos}

Agradecemos a Raulino Sabino da Silva e Carla Lourenço Tavares deAndrade(DAPS/EN SP) pela pesquisa no CNES e construção da amostra e a Gizene Luciana Pereira deSales (DAPS/ENSP) pela realização das figuras.

\section{Referências}

1. Porto SM, Santos IS, U gá AD. A utilização de serviços de saúde por sistemas de financiamento. Cien Saude Colet 2006;11(4):895-910.

2. Hulka B, Wheat J. Patterns of utilization: a patient perspective. M edical care 1985; 2395:438-460.

3. Ribeiro M CSA, Barata RB, Almeida M F, Silva ZP. Perfil sócio-demográfico e padrão de utilização de serviços de saúde para usuários e não-usuários do SUS - PNAD 2003. Cien Saude Colet 2006;11(4):10111022.

4. Lovalho A F. Administração de serviços de saúde em urgências e emergências. 0 mundo da saúde 2004; 28(2):160-171.

5. George S, Read S, Westlake L. Differences in priorities assigned to patients by triage nurses and by consultant physicians in accident and emergency departments. J Epidemiol Community $\mathrm{H}$ ealth 1993; 47(4):312-315.

6. Dale J, Green J. Primary care in the accident and emergency department: I. Prospective identification of patients. BMJ 1995; 12(311):423-426.

7. Gill JM, Riley AW. Nonurgent use of hospital emergency departments; urgency from patient's perspective. J Fam Pract 1996; 42(5):491-496.

8. Gill JM, Reese CL. Disagreement among health care professionals about urgent care needs of emergency. Ann Emerg M ed. 1996; 28(5):474-479.

9. Shah NM, Shah MA. Predictors of non-urgent utilization of hospital emergency services in Kuwait. Soc Sci M ed 1996; 42(9):1313-1323.

10. Hotvedt R, Thoner J, Wilskow AW, Ford HO. How urgent are emergency admissions? An evaluation of emergency admission to general hospitals in a Norwegian county. International Journal of Technology Assesment in Health Care 1999; 15(4):699-708.

11. Lee A, Lau FL, Hazlett CB. Factors associated with non-urgent utilization of accident and emergency services: a case-control study in Hong Kong. Soc Sci M ed. 2000; 51(7):1075-1085.

12. Coleman P, Irons R, Nicholl J. Will alternative immediate care services reduce demands for non-urgent treatment at accident and emergency? Emerg. M ed J. 2001; 18: 482-487.

13. Green, J, Dale, J. Primary care in accident and emergency and general practice; a comparison. Soc Sci Med 1992; 35(8):987-995.

14. M cGugan, $E A, M$ orrison, W. Primary care or $A \& E$ ? A study of patients redirect from accident $\&$ emergency departments. Scott M ed J 2000; 45(5):144-147.

15. Santos JS, Scarpelini S, Brasileiro SLL, Ferraz CA, Dallora MELV, Sá MFS. Avaliação do modelo de organização da unidade de emergência do HCFM RPUSP, adotando, como referência, as Políticas Nacionais de Atenção às U rgências e de Humanização. M edicina, Ribeirão Preto 2003; 36:498- 515.

16. Walt G. Health Policy. An Introduction to Process and Power. Johannesburg: Witwatersrand University Press; 1994.

17. Portal da Saúde. Projeto BRA 98/006 - edital 22/04. Disponível em: http://www.saude.gov.br 
18. Santos JS. Da fundação do Hospital das Clínicas à criação da Unidade de Emergência e sua transformação em modelo nacional de atenção hospitalar às urgências. M edicina, Ribeirão Preto 2002; 35:403-418.

19. Portaria GM/M S no 2.048 de 05 de novembro de 2002. Institui o regulamento técnico dos Sistemas Estaduais de U rgência e Emergência. Diário Oficial da União 2002; 12 nov.

20. Hartz ZMA, Contandriopoulos AP. Integralidade da atenção e integração de serviços de saúde: desafios para avaliar a implantação de um "sistema sem muros". Cad Saúde Pública 2004; 20(2):5331-5336.

21. Brasil. M inistério da Saúde. Carta dos Direitos dos U suários em Saúde, MS, 2006),

22. Fórum da Reforma Sanitária Brasileira. O SUS pra valer: universal, humanizado e de qualidade. Manifesto do Fórum da Reforma Sanitária Brasileira. Rio de Janeiro: CCI/ENSP; 2006. Disponível em : http:/ /www.abrasco.org.br

23. Giglio-Jacquemot A. U rgências e emergências em saúde: perspectivas de profissionais e usuários. Rio de Janeiro: Fiocruz; 2005.

24. Giddens A. A constituição da sociedade. São Paulo: $M$ artins Fontes; 1984

25. Costa EA, Rozenfeld S. M arcos históricos e conceituais. Constituição da Vigilância Sanitária no Brasil. In: Rozenfeld S, organizadora. Fundamentos da Vigilância Sanitária. Rio de Janeiro: Fiocruz; 2000. p.15-40.

26. Cochran W. Sampling Techniques. New York: John Wiley \& Sons; 1977.

27. Jacobs PC, M atos EP. Estudo exploratório dos atendimentos em unidade de emergência em Salvador - Bahia. Rev.Assoc.M ed. Bras. 2005; 51(6):348-353.

28. Furtado BMASM, Araújo Jr JLC, Cavalvanti P. O perfil da emergência do Hospital da Restauração: uma análise dos possíveis impactos após a municipalização dos serviços de saúde. Rev. Bras. Epidemio.2004; 7(3):270-289.

29. Pinheiro R. As práticas no cotidiano na relação oferta e demanda dos serviços de saúde: um campo de estudo e construção da integralidade. In: Pinheiro R, M attos RA, organizadores. Os sentidos da integralidade na atenção e no cuidado à saúde. Rio de Janeiro: Abrasco; 2001. p. 65-112.

30. Feliciano KVO, Kovacs MH, Sarinho SW. Sentimentos de profissionais dos serviços de pronto-socorro pediátrico: reflexões sobre o burnout. Rev. Bras. Saúde M ater. Infant. 2005; 5(3):319-328.

Artigo apresentado em 25/05/2006

Aprovado em 29/03/2007

Versão final apresentada em 24/04/2007 